\section{Cahiers de Narratologie}

Analyse et théorie narratives

$10.1 \mid 2001$

La voix narrative

\title{
La figure du lecteur dans les contes de Théodore de Banville
}

\section{Philippe Andrès}

\section{(2) OpenEdition \\ Journals}

\section{Electronic version}

URL: http://journals.openedition.org/narratologie/6917

DOI: 10.4000/narratologie.6917

ISSN: 1765-307X

\section{Publisher}

LIRCES

\section{Printed version}

Date of publication: 1 January 2001

Number of pages: $77-87$

ISBN: 2914561032

ISSN: 0993-8516

\section{Electronic reference}

Philippe Andrès, "La figure du lecteur dans les contes de Théodore de Banville", Cahiers de Narratologie [Online], 10.1 | 2001, Online since 16 October 2014, connection on 23 February 2021. URL: http:// journals.openedition.org/narratologie/6917 ; DOl: https://doi.org/10.4000/narratologie.6917 


\title{
LA FIGURE DU LECTEUR DANS LES CONTES DE THÉODORE DE BANVILLE
}

\author{
Philippe ANDRÈS \\ Université de Picardie
}

Il est doublement légitime de s'intéresser en ces lieux à Théodore de Banville. En effet, une approche biographique pourrait à elle seule justifier une lecture de l'auteur de La Mer de Nice'. Mais c'est l'énorme production en prose du poète considéré comme parnassien, qui va constituer l'objet de notre interrogation, et ce, essentiellement sous l'angle des contes publiés autour des années $1880-1890 \mathrm{chez}$ Charpentier ${ }^{2}$. Nous ne chercherons pas à tout prix à souligner l'originalité de l'écriture banvillienne, quoique le fait même de continuer à publier des recueils poétiques tout en exerçant la tâche de feuilletoniste (chroniqueur, critique, conteur) dans les journaux, soit déjà la marque d'une volonté de briser les séparations entre les genres. Nous ne parlerons pas non plus du lecteur historique, celui de la réception contemporaine ou non de l'œuvre en question. Nous nous proposons simplement de souligner l'importance du recours quasi systématique voire rhétorique à la figure du lecteur, qui se présente sous de multiples formes, mais qui toutes ont en commun le souci de ne pas ennuyer.

Le lecteur n'est pas un personnage comme un autre puisque, en dehors de ses présences en tant que tel dans le texte, il est " une figure virtuelle: le destinataire implicite auquel le discours s'adresse ${ }^{3}$. Dans les contes banvilliens, il apparaît

1 Théodore de BANVILLE, La Mer de Nice. Lettres à un ami, Paris, Poulet-Malassis et de Broise, 1861.

2 Philippe ANDRÈS, "Banville et l'art du conte ", NineteenthCentury French Studies, Vol. 24, 1996, p. 381-387.

3 Vincent JOUVE, La lecture, Hachette, Supérieur, 1997, p. 24. 
très souvent avec le statut de "narrataire invoqué ${ }^{4}$, et notamment dans des lieux stratégiques (ouverture ou fermeture narratives) selon le procédé rhétorique formant cliché de l'apostrophe mimant la parole orale. Dans l'incipit, il joue un rôle cataphorique, et se présente la plupart du temps sous la forme pronominale de la seconde personne du pluriel. Cela est bien commode, puisque le « vous » efface la référence à un personnage précis, le vide de son contenu, pour l'auréoler d'une proximité fictive. Ainsi, dans les Contes hérö̈ques, la voix narrative conteuse, dès les premiers mots se substitue à une parole magique :

Imaginez un jeune homme de vingt-quatre ans, beau, robuste, exempt de soucis, qui se promenant dans Paris par une matinée de mai [...] se trouve en moins de cinq minutes avoir ressenti toutes les extases de l'amour et toutes les angoisses du plus profond désespoir ! Marcel Pauli marchait $[\ldots]^{5}$.

De cette manière la distance entre narrateur et narrataire semble s'abolir, faisant du lecteur une sorte de projection virtuelle, un alibi, un prétexte à dire le conte. Et ce dialogue supposé qui prend les allures d'une proximité verbale cache un rapport de force implicite où triomphe le narrateur qui n'hésite pas à s'impliquer à la première personne du singulier :

Le lecteur voudrait sans doute un nom plus poétique, et je n'aurais pas manqué de le choisir tel, s'il m'eût été permis d'inventer $[\ldots]^{6}$.

Ici, le narrateur se substitue littéralement au désir du lecteur (emploi de la forme en -rais, et du modalisateur à valeur hypothétique) qui n'est qu'un faire-valoir rhétorique de la narration. La voix narrative se réfugie derrière la véracité de ce qui se raconte, comme si le recours à l'expérience vécue avait valeur universelle. Or, l'incipit de ce conte insiste sur la différence entre une époque heureuse (celle du théâtre populaire

4 Vincent JOUVE, op. cit., p. 27.

5 Théodore de BANVILlE, Contes hérö̈ques, Scènes de la vie, « Jeannette ", Charpentier, 1884, p. 179.

6 Théodore de BANVILLE, Esquisses parisiennes, Scènes de la vie, « La vie et la mort de Minette », Charpentier, 1876, p. 265. 
de la Restauration) et un aujourd'hui (celui de l'énonciation) qui paraît fustiger le théâtre naturaliste :

Autant les auteurs cherchent aujourd'hui à atteindre une réalité d'où puissent découler des enseignements, autant alors, se bornant au rôle modeste d'étourdir et de distraire au lieu d'instruire, ils employaient tous leurs efforts à faire vivre le spectateur au milieu des plus étincelantes poésies du rêve [...] ${ }^{7}$.

On peut facilement constater que l'appel rhétorique à la figure lectorale se transforme en justification idéologique voire en regret envers une époque révolue où théâtre et fantaisie allaient de pair. Et l'incipit n'en finit pas de prendre un détour pour légitimer le récit qui va suivre. De même, à la fin de ce conte, le narrateur insiste habilement sur l'enracinement de son récit dans le monde réel :

Et maintenant, avant d'écrire les dernières lignes de cette histoire, (car le dénoûment en fut trop horrible pour ne pas devoir être raconté en quelques mots,) j'ai besoin de rappeler au lecteur que c'est la réalité elle-même qui nous montre certaines existences vouées tout entières à une infortune imméritée et implacable ${ }^{8}$.

Cette insistance nous semble la marque d'une intentionnalité didactique à but moralisateur, comme si la destinée de Minette devait s'auréoler d'un caractère exceptionnel, celui du «martyr ». Ainsi le discours qui revendique une réalité hors du commun et en même temps banale ne peut être pris au pied de la lettre. La parole conteuse se perd dans une aporie, une sorte de double bind que souligne le recours trop appuyé au lecteur. Le conteur ne cesse de porter un regard critique sur sa narration :

D'abord je répondrais que j'essaye de raconter et non pas d'expliquer cette douce et poétique folie ; mais n'y aurait-il pas là le sujet d'une remarquable étude psychologique? Une fois notre éducation faite, nous ne nous rappelons pas assez les peines qu'on s'est données pour séparer dans notre esprit le merveilleux du réel ; nous

7 Théodore de BANVILLE, op. cit., p. 258.

8 Théodore de BANVILLE, op. cit., p. 312. 
oublions tout ce qu'il a fallu d'études, de raisonnements et d'expériences pour détruire en nous cette confusion qui enivre les âmes naïves $[\ldots]^{9}$.

Cette présence métacritique narrative est suspecte. Sommesnous encore dans un conte ? Tout se passe comme si cette présence hypertrophiée du narrateur était la marque d'une oralité mimée mais également minée de l'intérieur. En effet, il ne s'agit plus de relancer simplement l'intérêt du lecteur (relance appartenant à la stratégie de la parole conteuse), mais plutôt de proposer une réflexion du lecteur sur ce que raconter veut dire, qui prend les allures d'une proximité confidentielle (recours à la première personne du pluriel englobant le lecteur dans une même expérience vécue). C'est pourquoi la magie du conte disparaît au profit d'une autojustification de la narration. En vain, semble-t-il, Banville tente-t-il de rénover le genre ... Le dialogue fictif cache en fait un monologue réflexif à la fois métacritique et rhétorique. Le lecteur se transforme en une figure-prétexte.

Situé à la fin du conte, le "narrataire-invoqué » n'est pas qu'un simple outil de rappel, de relance rhétorique interne au récit. Il peut également faire partie d'une démarche purement économique : il s'agit de faire en sorte que le lecteur achète le journal, la fois suivante.

Par ailleurs, nombreux sont les récits banvilliens qui traitent du théâtre, milieu que notre auteur connaît bien. Et l'on peut assister à une sorte de théâtralisation dans la relation narrateur-lecteur, comme si le sujet du récit et la manière de raconter s'inscrivaient dans un rapport métaphorique. Dès la parution des Pauvres saltimbanques en 1853, Banville rend vivant son récit en plongeant le lecteur dans une représentation feinte, quand il dresse, par exemple le portrait de Frédérick Lemaître :

Celui-là, regardez-le, écoutez-le ; quel spectacle ! Il frémit et vibre sans cesse comme le violon de Paganini ; sublime ou ridicule, à toute heure un enthousiasme

9 Théodore de BANVILLE, op. cit., p. 277. 
quelconque fait frémir sa lèvre, ouvre sa narine, raidit sa crinière de lion ${ }^{10}$.

Outre l'interpellation à la deuxième personne du pluriel (notons l'importance de l'effacement du mot « lecteur») qui met la scène sous les yeux du lecteur, le narrateur utilise le présent à valeur d'hypotypose. Nous avons l'impression d'être là et d'assister en direct au portrait en chair et en os du célèbre acteur. Le lecteur est convoqué dans l'abolition d'une distance, mais au cœur d'une rhétorique où le chiasme crée un rebondissement. C'est la narration elle-même qui devient spectaculaire, qui reflète et se donne à voir dans une sorte de jouissance que maîtrise le narrateur. Le récit se théâtralise et épouse ainsi l'objet de son dire. Or, il existe plusieurs degrés dans cette théâtralisation narrative, qui, audelà des conventions, instaure un rapport particulier avec le lecteur.

Ainsi, une des originalités banvilliennes, semble-t-il, consiste à féminiser la figure du lecteur. Le recueil des Contes pour les femmes en est la parfaite illustration, non seulement dans le titre lui-même, mais dans les termes mêmes de l'avant-propos que l'on trouve dans l'édition de $1881 \mathrm{chez}$ Charpentier, mais qui disparaît dans la réédition chez Arthème Fayard", sans doute parue dans la première moitié du $\mathrm{XX}^{\mathrm{e}}$ siècle, entre les deux guerres. Dans le dernier paragraphe de cet avantpropos, Banville justifie la féminisation de son destinataire :

Les Femmes reconnaîtront bien que ces Contes ont été écrits pour elles, car j'en ai banni avec soin toutes les vieilles conventions banales, que d'ordinaire on prétend leur faire accepter comme articles de foi. J'ai cru que la vertu et l'amour sont des choses assez grandes en ellesmêmes pour se passer des hypocrites mensonges et des fictions sentimentales.

10 Théodore de BANVILLE, Les pauvres saltimbanques, « Frédérick Lemaître ", Paris, Michel Lévy, Frères, p. 77.

11 Cette édition, sans date, Paris, Modern-Bibliothèque, Illustrations d'après les dessins de Paul Allier, remplace cet avant-propos ainsi que le "Dizain pour commencer " et la "ballade pour finir " de l'édition Charpentier, par une abondante iconographie dans le style Arts-décos où les représentations féminines jouent un rôle très important. 
L'incitation rhétorique à la lecture se veut ici dénuée de toute rhétorique, ce qui semble paradoxal étant donné la fonction stratégique de tout avant-propos. Cependant, sous couvert d'une tonalité humoristique, l'objectif de l'auteur est clairement annoncé : faire du neuf et briser les habitudes anciennes. D'emblée, la narration banvillienne cherche à s'inscrire dans une modernité visant un public délibérément choisi. S'agit-il encore de rhétorique, ou bien, comme nous inclinons à le penser, d'une lucidité de la part de notre auteur pour toucher un lectorat nouveau et qui tend à prendre une place de plus en plus grande ? Ce procédé d'ailleurs se retrouve, sans pour autant être systématique (car la diversité est l'un des charmes de l'œuvre banvillienne !) dans l'avant-propos de $L a$ Lanterne magique, comme un souci constant de montrer de l'intérêt pour le destinataire ${ }^{12}$, d'autant plus que ce recueil est suivi d'un dizain « Aux lectrices»!

La théâtralisation de l'acte de lecture se situe au cœur même de la problématique narrative. En effet, le recueil des Contes pour les femmes se trouve encadré par un "dizain, pour commencer » et une "ballade pour finir». Il s'agit d'un procédé équivalant au prologue et aux remerciements adressés au public que l'on trouve dans une certaine tradition théâtrale que Banville a par ailleurs mis en pratique ${ }^{13}$. Intégrés au recueil, les poèmes à forme fixe, outre leur intérêt d'annonce et de clôture par rapport aux soixante contes, fonctionnent comme une justification de l'œuvre tout entière. En effet, il s'agit d'un clin d'œil au lecteur qui est censé avoir lu, entre autres, les Trente-six ballades joyeuses publiées en 1873. Voilà donc un rappel de la création poétique antérieure

12 La Lanterne magique, "Petites Etudes », Charpentier, 1883. "Quant à la femme, le seul moment dont elle puisse disposer en faveur de la littérature, c'est les deux minutes pendant lesquelles sa femme de chambre lui met ses bas, comme en témoigne le spirituel dessin de Georges Rochegrosse placé en tête de ce volume. "

13 On songe notamment aux prologues écrits par Banville, comme celui des Folies Nouvelles, le 21 octobre 1854 ; son Impromptu «Petit prologue en vers ", dit pour l'ouverture du Théâtre du Palais Royal, le 14 septembre 1880 , ou Le messager, "Prologue d'ouverture ", à l'Odéon, le 15 septembre 1880. 
du conteur, comme une sorte de publicité discrète, d'autant plus que l'annonce publicitaire parue au dos du recueil est sans ambiguïté 14 . Cet encadrement transforme le statut du lecteur qui est à la fois auditeur supposé d'un conte oral ; lecteur du texte écrit et connaisseur supposé de l'œuvre banvillienne: cette mise en scène souligne la visée intentionnelle de l'auteur par rapport à un public choisi.

Ce procédé de féminisation du destinataire se retrouve systématiquement dans l'œuvre narrative banvillienne. Dans les Camées parisiens nous retrouvons, outre le dialogue intertextuel essentiel avec les Emaux et Camées de Gautier, une interrogation esthétique majeure concernant les liens entre narrativité et représentation picturale, et notamment l'utilisation d'une forme brève éclatée: le médaillon. Chaque douzaine s'ouvre sur une interpellation au public féminin et met en scène une "galerie de portraits parisiens » métaphorisée par l'image désormais cliché de la transformation du " caillou » en " pierre précieuse »15. Cette volonté d'esthétiser le petit format, le modèle réduit, élève au rang d'œuvre d'art la miniature narrative ou picturale, et la tonalité ludique cache à peine une réflexion sérieuse sur les limites de l'œuvre accomplie :

Certes, dans cette galerie de poche, il manque bien des profils parisiens absolument célèbres et d'une importance indiscutable ; mais ce qui caractérise nos œuvres, c'est surtout ce qu'elles ne contiennent pas, et le propre de tous les travaux humains, c'est d'être incomplets; car cette vie terrestre n'est qu'une gare, que les artistes

14 «Les Scènes de la Vie où Théodore de Banville se révèle comme un conteur d'une observation intense et nouvelle, ne le cèdent en rien pour l'originalité de son inspiration à son oeuvre poétique [..] ». Voir P. Andrès ; "Banville et l'art du conte ", Nineteenth-Century French Studies, Vol. 24, 1996, p. 381-387.

15 «Mais cette fois encore, j'espère que votre bonté magique, trèspuissante et mère de tous les miracles, suppléera à l'insuffisance de l'ouvrier, et qu'en touchant seulement ces cailloux mal égratignés par mon outil, vos petites mains transparentes et vos doigts de fées en feront des pierres véritablement précieuses !", Théodore de Banville, Camées parisiens, « Les introuvables », 1994, p. 42. 
traversent en cherchant de belles concordances de lignes, et les Anglais en tenant leur couverture de voyage, pliée et roulée dans une courroie $[\ldots]^{16}$.

Il ne s'agit pas d'une rhétorique facile à destination d'une lectrice bienveillante et gagnée d'avance par la tonalité humoristique, mais bien plutôt d'une réflexion sur la fonction de l'art en général. Cependant, le médaillonniste veut éviter le piège de la théorisation afin de ne pas ennuyer le destinataire. La lecture, malgré les déchirements de la guerre francoprussienne (la dernière douzaine est datée de décembre 1872), s'inscrit délibérément dans une tonalité ludique, résultat d'une volonté de dépassement de l'événement. Il ne s'agit pas de nier le rôle de l'histoire et de ses déchirures, mais de transformer et de maintenir une joie créatrice, dans un rapport de proximité amusée avec la lectrice qui ne doit pas être dupe de la badinerie apparente. Ce qui est en jeu, c'est une interrogation sur l'acte de narrer et l'implication du destinataire.

Or, cette capacité conteuse qui nécessite une stratégie narrative théâtralisée se retrouve dans une publication tout à fait intéressante et originale à laquelle Théodore de Banville non seulement a collaboré, mais a participé à une place de choix : je veux parler du recueil du Nouveau Décaméron ${ }^{17}$, pastiche générique et thématique des œuvres de Boccace et de Marguerite de Navarre. Le " roi de la première journée » est Théodore de Banville dont le médaillon orne le recueil avec celui de Cypris Aphrodite : s'ensuit la liste des neuf autres conteurs. L'avant-texte souligne l'originalité de ce Décaméron " écrit » au lieu d'être "parlé », et également le fait de taire la véritable identité des «belles écouteuses ", comme une sorte de pudeur, alors que le conteur-narrateur avance une signature connue des autres personnages. Cette invitation à la découverte des clés participe du plaisir de la lecture, sous l'égide de la marquise Thérèse de Lionne qui raconte sa propre histoire quelque peu égrillarde, donnant ainsi le ton de l'ensemble du recueil.

16 Camées parisiens, op. cit., p. 161.

17 Le Nouveau Décaméron, Première Journée «Le temps d'aimer ", Paris, E. Dentu, 1884. 
Dans le cadre aristocratique d'un château avoisinant Versailles, et le temps pluvieux donnant l'occasion de dire des contes, la conversation s'engage et l'on part à la recherche d'un "premier souverain». Chaque recueil s'inscrit sous l'autorité d'un roi et d'une thématique. Théodore de Banville s'esquive, prétextant "l'idée de fumer une cigarette ", mais rattrapé, l'" auteur des Exilés, ne peut échapper à la royauté : sa reine «olympienne et parisienne à la fois » tiendra de la mythologie grecque (Cypris) et de la pantomime (Colombine) : Madame d'Albereine au nom prédestiné ! Le conte inaugural dit par Banville le lendemain, s'intitulera : «La vie en rêve » 18 , et mettra en scène la destinée tragique d'une jeune fille qui se meurt, " dévorée par un mal invisible et profond ». Ce récit ne semble pas sans rapport avec $L e$ Rêve d'Emile Zola, publié quatre ans plus tard (présence redondante du thème de la blancheur, similitude du titre, ...). Il n'est pas inintéressant, d'un point de vue génétique, de postuler une influence intertextuelle banvillienne. Emile Zola d'ailleurs, apparaît pour la première fois au cours de la troisième journée («Les Amours mondaines ») pour un conte intitulé « Le jeûne ». C'est le seul récit du recueil orné d'une gravure montrant le prêche du vicaire ${ }^{19}$. Le romancier naturaliste deviendra le roi de la cinquième journée ( La rue et la route »), ouvrant ainsi le recueil par le récit de «La mort d'un paysan ${ }^{20}$. De cette curiosité fictionnelle, il convient de retenir non seulement le jeu avec le modèle de Boccace, mais aussi la présence quotidienne de Théodore de Banville, reconnu comme conteur, comme maître de la narration écrite, par ses pairs, alors que ce dernier est de nos jours célèbre en tant que poète.

Ce lien étroit entre le narratif et le poétique nous paraît essentiel pour comprendre non seulement une œuvre méconnue, mais aussi pour poser le problème de la relation

18 Le Nouveau Décaméron, op. cit., p. 29-40.

19 Il serait intéressant de considérer ce texte en relation avec $L e$ Rêve qui sera publié en 1888 .

20 Le Nouveau Décaméron, Cinquième journée, "La mort d'un paysan ", p. 11 . 
entre les genres dans la seconde moitié du XIX ${ }^{\mathrm{e}}$ siècle. Les clivages ne sont plus étanches; les zones frontalières semblent s'estomper, sans doute au profit du narratif par nécessité de s'adapter aux goûts et aux conditions de lecture de l'époque. Les contemporains de Banville ont lu son œuvre dans sa complexité poétique et narrative. Et nous, nous occultons cette dimension, comme si la part narrative était dotée d'on ne sait quel coefficient négatif, résolus que nous sommes à ne voir de l'œuvre intégrale que la part poétique, voire parnassienne !

Toute activité narratrice postule une relation ludique et implique un minimum de connivence avec le lecteur qui peut ou non (en restant hors-jeu) participer de cet appel au jeu, comme nous l'a rappelé Michel Picard :

Subversion dans la conformité, élection du sens dans la polysémie, modélisation par une expérience de réalité fictive, ces critères désignent en réalité trois fonctions, auxquelles seul le cadre du jeu permet de donner toute leur valeur et leur profonde convergence. Encore faut-il savoir, et pouvoir, jouer ${ }^{21}$.

Ce jeu banvillien avec la figure du lecteur, jeu éminemment sérieux puisque s'inscrivant dans une lucidité esthétique transgénérique, nous renvoie à notre propre image. Désormais, il n'est plus possible de rejeter comme dépourvue de valeur, la part du ludique qui appartient, selon nous, au projet de l'écrivain. Affiner notre esquisse par une recherche systématique des occurrences des différentes figures du lecteur nous paraît une problématique digne d'intérêt sur le plan herméneutique, si elle ne se limite pas à une typologie purement formelle. En effet, tiraillé entre un «sujet jouant » et un "sujet joué » (un liseur et un $l u$ dans la terminologie de Picard) ${ }^{22}$, le lecteur-

21 Michel PICARD, La lecture comme jeu, Les Editions de Minuit, 1986, p. 266.

22 Michel PICARD, op. cit., «Le jeu dédouble celui qui s'y adonne en sujet jouant et sujet joué : ainsi y aurait-il un liseur et, si l'on ose dire, un lu. Le joué, le lu, seraient du côté de l'abandon, des pulsions plus ou moins sublimées, des identifications, de la re-connaissance et du principe de plaisir [...]. Le sujet jouant, le liseur, seraient du côté du 
critique - le «lectant»- cherche inlassablement à se dépouiller des a priori du vieil homme et des enthousiasmes du découvreur, et considère l'œuvre dans sa singularité. Banville prosateur nous surprend dans notre torpeur dogmatique et nous invite à lire, autrement, les phares de la narration du XIX ${ }^{\mathrm{e}}$ siècle.

réel, les pieds sur terre, mais comme vidés d'une partie d'eux-mêmes, sourdes présences [...], p. 112. 